\title{
A case of two siblings with Morquio syndrome
}

\author{
Kankananarachchi I ${ }^{1}$, Amarasena $\mathrm{S}^{1}$ \\ ${ }^{1}$ Faculty of Medicine, University of Ruhuna, Sri Lanka
}

\section{Abstract}

Morquio syndrome (Type IV mucopolysaccharidosis) is an autosomal recessive condition with a prevalence of 1:40000 to 1:200000.1 The deficiencies of N-acetyl-galactosamine-6-sulfatase and beta-galactosidase leads to accumulation of glycosaminoglycan resulting Morquio syndrome type A and B respectively. Here, we report a case of two siblings with Morquio syndrome. Parents brought two male siblings aged 5 years and 30 months due to abnormal physical appearance. They were born to non-consanguineous healthy parents who had uneventful antenatal periods. Parents were worried about short stature and abnormal chest shape. On examination, both of them had similar physical characteristics such as a coarse face, short stature, pectus carinatum, scoliosis, short neck, coxa vulga, and multiple bony deformities. They had normal basic biochemistry, haematological indices, and bone profil . Thoracolumbar spine and pelvis $\mathrm{X}$ rays showed platyspondyly with anterior beaking and flaring of iliac wings. Ophthalmology assessment of both siblings revealed amblyopia. Both siblings had elevated urine glycosaminoglycans. Qualitative urine analysis showed moderate excretion of chondroitin sulphate and mildly elevated Heparan sulphate, which favoured MPS type IV. Lysosomal enzyme assay performed on elder sibling showed a low level of beta-galactosidase-6-Sulphate-Sulphatase and normal level of beta-galactosidase, and the diagnosis was confi med as MPS type IV-A. Since no curative therapy was available yet, parents were explained about the disease condition, and follow-up was arranged with a multidisciplinary approach. Unfortunately, there is a significant amount of financial constraint in diagnosing and managing Morquio syndrome. Developing countries such as Sri Lanka cannot afford enzyme replacement therapy, and HSCT is not developed for children with inborn errors of metabolism. However, it is essential to have surveillance for anticipated complications of the condition with a multidisciplinary team approach until a cheaper disease-modifying agent is available.

Key Words: primary aldosteronism, adrenal venous sampling, unilateral catheterization

\section{Correspondence email: imalke462@gmail.com}

https://orcid.org/0000-0002-9351-2966

This is an open-access article distributed under the terms of the Creative Commons Attribution License, which permits unrestricted use, distribution, and reproduction in any medium, provided the original author and source are credited (CC BY 4.0)

\section{Introduction}

Morquio syndrome (Type IV mucopolysaccharidosis) is an autosomal recessive condition with a prevalence of 1:40000 to 1:200000.1 The deficiencies of N-acetyl-galactosamine-6-sulfatase and beta-galactosidase leads to accumulation of glycosaminoglycan resulting in Morquio syndrome type A and
B respectively (1). Here, we report a case of two siblings with Morquio syndrome.

\section{Case Report}

Two siblings, 5 years old boy (Case 1, figure 1) and 2 years and 6-month old boy (Case 2, figure 2) were brought by parents due to abnormal physical ap- 
pearance. They were born to non-consanguineous healthy parents who had uneventful antenatal periods. Parents were worried about short stature and abnormal chest shape. On examination, both of them had similar physical characteristics such as a coarse face, short stature, pectus carinatum, scoliosis, short neck, coxa vulga, and multiple bony deformities. Both of them had normal intellectual development.

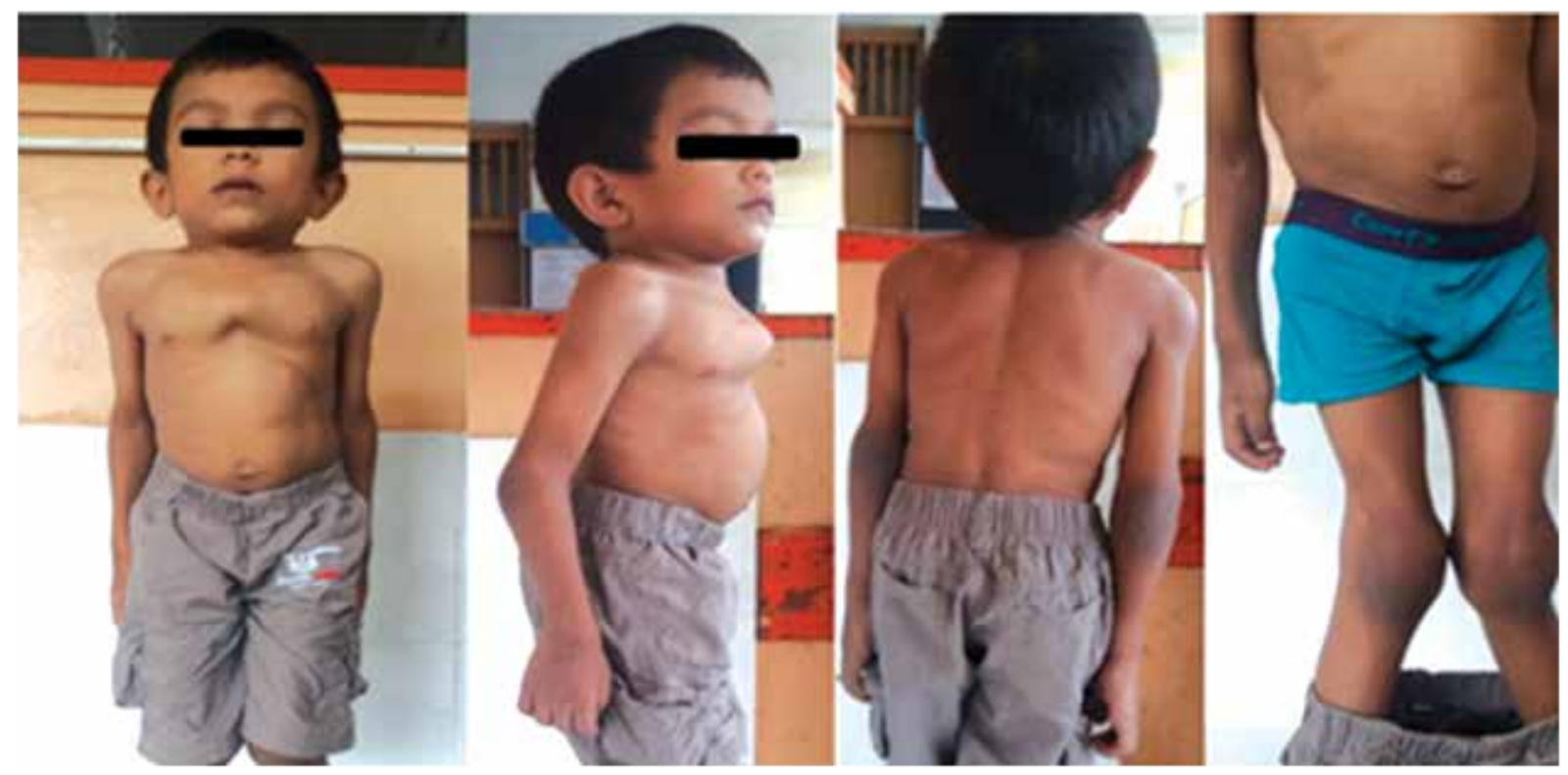

Figure 1 Five-year-old boy with Morquio syndrome

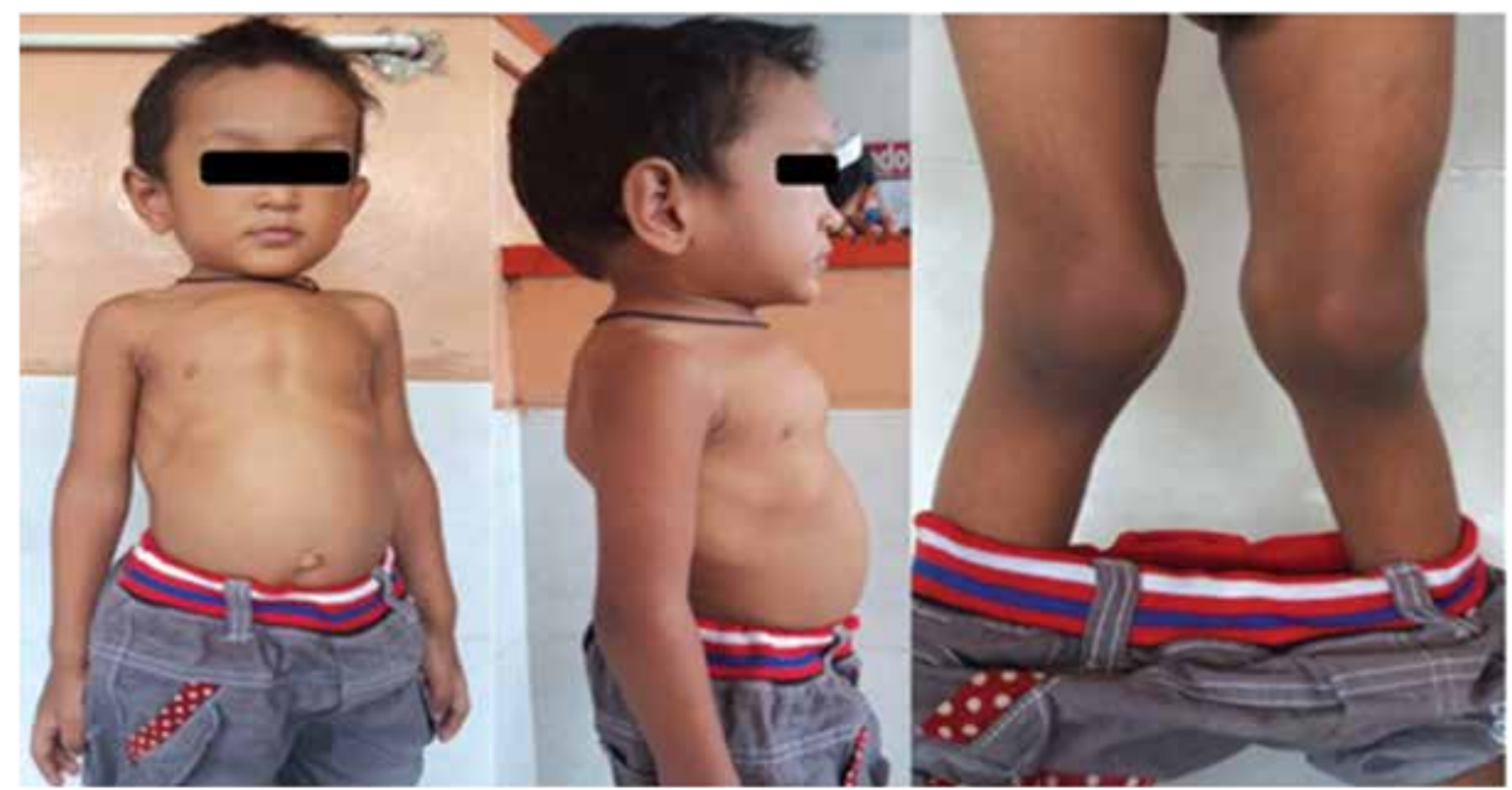

Figure 2 Three-year-old boy with Morquio syndrome

Both siblings had normal basic biochemis- both siblings showed flat ened vertebral bodies try, hematological indices, and bone profil . (platyspondyly) with anterior beaking and flaring of Thoracolumbar spine and pelvis X-rays of iliac wings (Figure 3). 


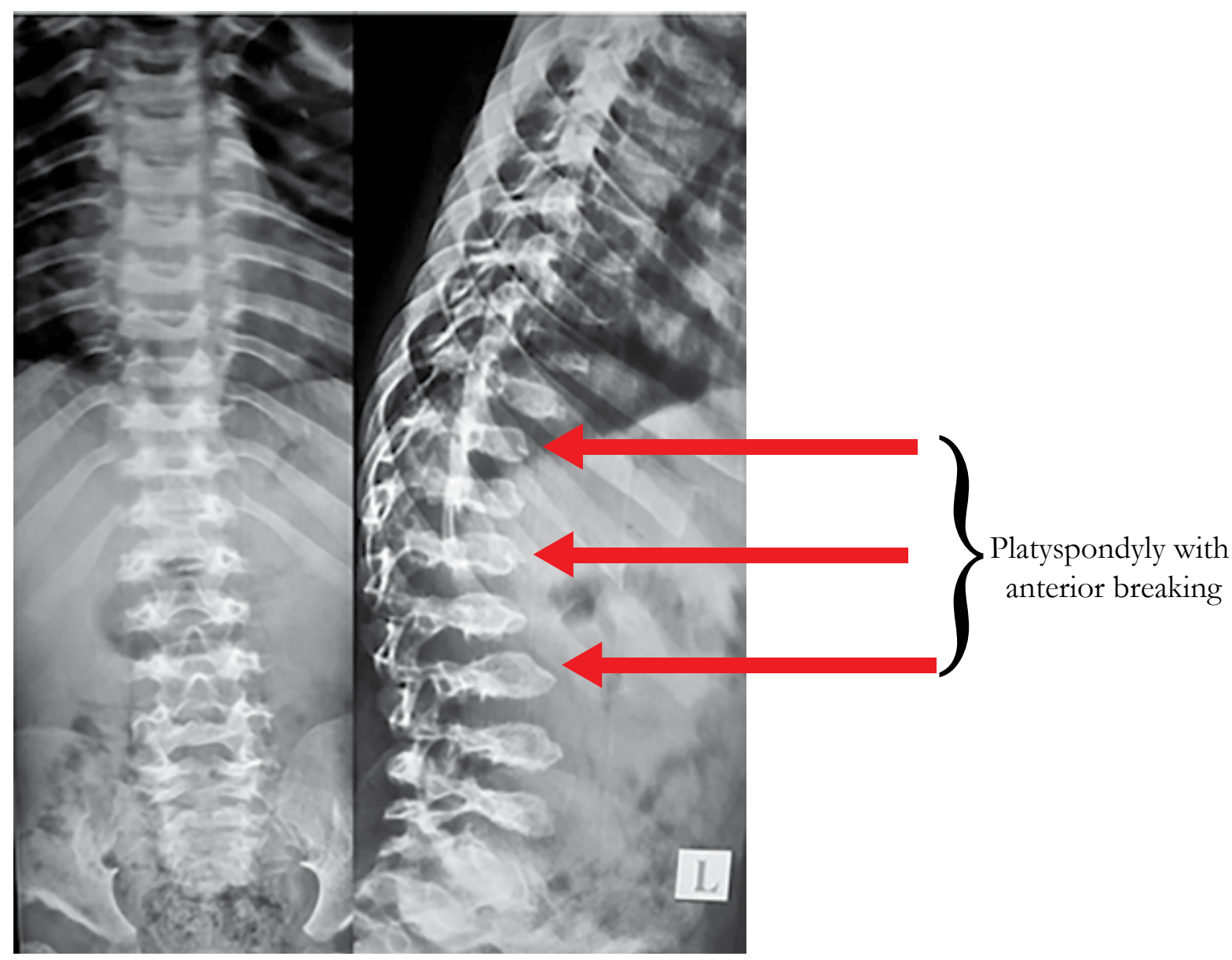

Figure 3 X-ray thoracolumbar spine

Their hand X-ray revealed broad meta- pointed proximal metacarpals of second and fifth carpals, irregular carpal bones, and fin ers (Figure 4).

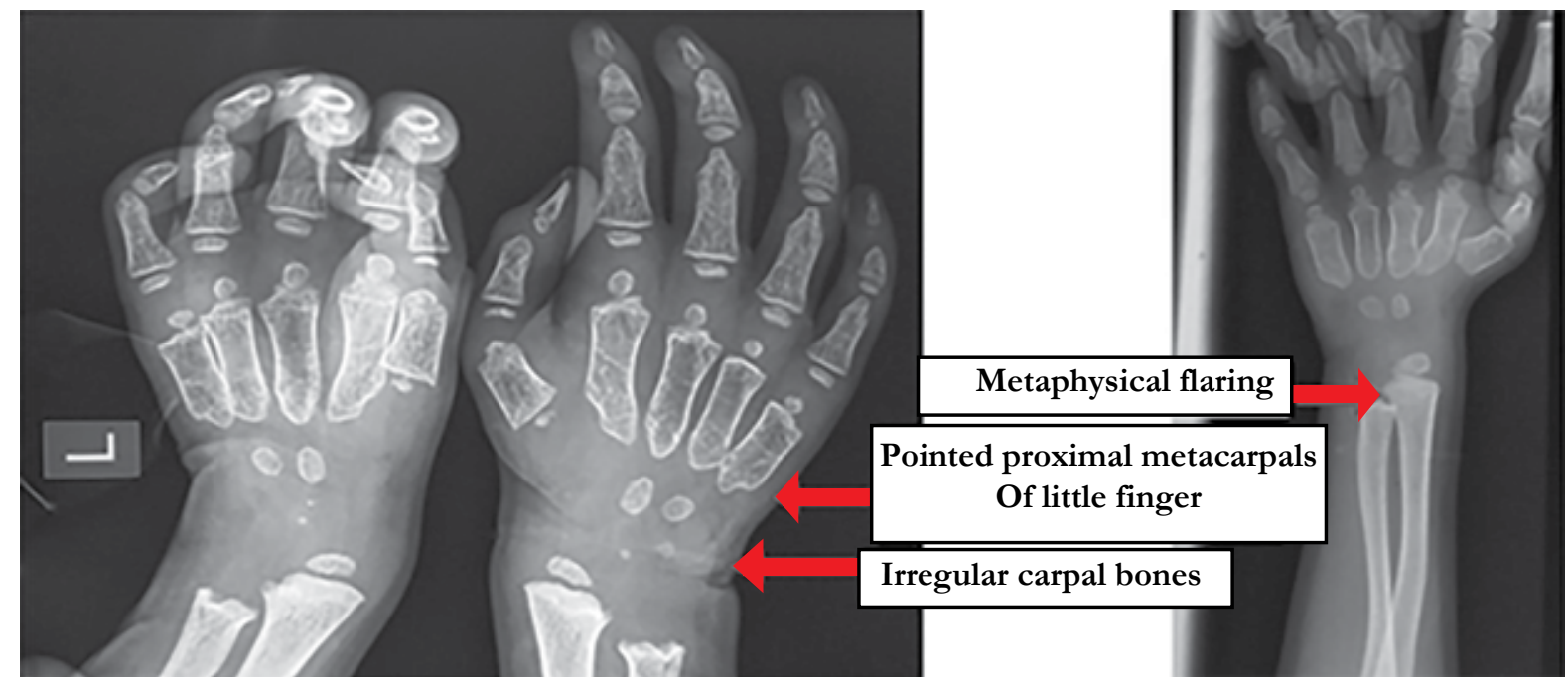

Figure 4 Hand X rays showing carpal and metacarpal bone changes 
Due to the clinical suspicion of Mucopolysaccharidosis (MPS), the siblings underwent testing for urine for Glucosamine glycan (GAG) and they had elevated urine for GAG. Qualitative urine analysis showed moderate excretion of chondroitin sulfate and mildly elevated Heparan sulfate which was more in favor of MPS type IV. The lysosomal enzyme assay was performed in the elder brother's blood and showed a low level of beta-galactosidase-6-Sulphate-Sulphatase, $6.2 \mathrm{nmol} / \mathrm{hrs} / \mathrm{mg}$ protein (14 32). and normal level of beta-galactosidase, 210.1 nmols/hrs/mg protein (79.6-480.0). Based on the findings of enzyme assay the diagnosis of Morquio A (MPS type IVA) was made on an elder sibling. The lysosomal enzyme assay was not done on the younger brother at the time of publication.

Ophthalmology assessments of both siblings revealed amblyopia and they were prescribed on spectacles. The hearing assessments did not reveal any deficiency. There was no abnormality found in the $2 \mathrm{D}$ echocardiographs. Since no curative therapy was available yet, parents were explained about the disease condition and follow-up was arranged with a multidisciplinary approach.

\section{Discussion}

Morquio syndrome A is secondary to the accumulation of Keratan sulfate and chondroitin Sulphate in the Extracellular matrix and cartilages due to a defect in the metabolism of glycosaminoglycan (2). The defi ient enzyme in MS is N-acetyl-galactosamine-6-sulfatase and the genetic mutation occurs on chromosome 16q24.3 (2). Preservation of intelligence and short trunk dwarfisms are the two distinctive features of MS compared to other MPS. MPS IV-A is more severe compared to MPS-IVB with an average adult height of around $125 \mathrm{~cm}$ and $150 \mathrm{~cm}$ respectively (3).

Skeletal manifestations of MPS IV-A are coxa vulgar, short stature, kyphosis, pectus carinatum, and short neck. Instability of the odontoid process is very common and can lead to paraplegia in later life. Corneal clouding, glaucoma obstructive airway disease, dental anomalies, and valvular heart lesions are common extra skeletal malformations of the disease (3). Skeletal abnormalities manifest earlier than other organ involvement and that would have been the reason for these siblings to not have significant extraskeletal manifestations yet.

Characteristic axial radiological findings of MPS IV-A include Platyspondyly, anterior beaking, atalnto axial subluxation, and goblet-shaped iliac wings. Metaphyseal flaring in long bones, irregular carpal bones, pointed metacarpals of the 5 th fin er, and short and wide tubular bones are some of the peripheral radiological features in MPS IV-A (3). Most of these radiological features were there in these two patients.

The initial clue for the diagnosis of MPS IV-A is elevated urine GAG and elevated KS in blood. Confi mation of the diagnosis needs a lysosomal enzyme assay. Genotyping is optional and is mainly used in genetic counseling (4).MPS IV -A can be treated with enzyme replacement therapy (ERT), Elosulfase alfa. However, the annual cost is around $\$ 380,000$ per patient (4). Hematopoietic stem cell transplant has also been an established treatment modality (5). Substrate reduction therapy using $\mathrm{fl}$ vonoid compounds, anti-inflammatory medications such as Infliximab and Gene therapy are still under the research level. All patients should be managed with a multidisciplinary approach involving, orthopedic, cardiology, ophthalmology, respiratory, maxillofacial, and otolaryngology teams. In the absence of newer treatment modalities, these two patients were given a multidisciplinary follow-up plan.

\section{Conclusions}

There is a significant amount of financial constraints in diagnosing and managing Morquio syndrome. Developing countries such as Sri Lanka cannot afford ERT and HSCT is not developed for children with inborn errors of metabolism. However, it is important to have surveillance for anticipated complications of the condition with a multidisciplinary team approach until a cheaper disease-modifying agent is available.

\section{Acknowledgment}

The authors would like to acknowledge Dr. Eresha Jasinghe, and the Department of Radiology, 
TH Karapitiya to facilitate diagnostic testing of the child.

\section{References}

1. Eggli KD, Dorst JP. The mucopolysaccharidoses and related conditions. In Seminars in roentgenology 1986 Oct 1; 21(4); 275-294. WB Saunders. doi. org/10.1016/0037-198X(86)90039-8

2. Kliegman, Stanton, St Geme Schor, Nelson Textbook of Paediatrics. $20^{\text {th }}$ Ed. Philadelphia, PA: Elsevier, 2016. Chapter 88: Mucopolysaccharidoses; p. 742

3. Montaño AM, Tomatsu S, Gottesman GS, Smith M, Orii T. International Morquio A Registry: clinical manifestation and natural course of Morquio A disease. Journal of Inherited Metabolic Disease:
Official Journal of the Society for the Study of Inborn Errors of Metabolism. 2007Apr;30(2):165-74. doi. org/10.1007/s10545-007-0529-7

4. Tomatsu S, Yasuda E, Patel P, Ruhnke K, Shimada T, Mackenzie WG, Mason R, Thacker MM, Theroux M, Montaño AM, Alméciga-Díaz CJ. Morquio A syndrome: diagnosis and current and future therapies. Pediatric endocrinology reviews: PER. 2014 Sep;12(0 1):141.

5. Yabe H, Tanaka A, Chinen Y, Kato S, Sawamoto K, Yasuda E, Shintaku H, Suzuki Y, Orii T, Tomatsu S. Hematopoietic stem cell transplantation for Morquio A syndrome. Molecular genetics and metabolism. 2016 Feb1;117(2):84-94. doi.org/10.1016/j. ymgme.2015.09.011 